\title{
A SIMPLE TRIANGULATION METHOD FOR SMOOTH MANIFOLDS ${ }^{1}$
}

\author{
BY STEWART S. CAIRNS \\ Communicated by Raoul Bott, March 23, 1961
}

We first triangulate a compact closed $m$-manifold $M^{m}$ of differentiability class $C^{r}(r>1)$ in a euclidean space $E^{\nu}=E^{m+n}$. The method is simpler than earlier methods (see References) and is applicable to a wider class of spaces (see $(F)$ and $(G)$ below).

(A) For a given $\eta>0$, let $\left(a_{1}, \cdots, a_{\mu}\right)$ be a set of distinct points on $M^{m}$ such that each point of $M^{m}$ is at distance $<\eta$ from at least one point $a_{i}$.

Let $d$ be the euclidean distance function in $E^{v}$. For each $k \in(1, \cdots, \mu)$, let

$$
\begin{aligned}
& \bar{c}_{k}^{\nu}=\left\{x \in E^{\nu} \mid d\left(a_{k}, x\right) \leqq d\left(a_{i}, x\right), \quad i=1, \cdots, \mu\right\}, \\
& \bar{\gamma}_{k}^{m}=M^{m} \cap \bar{c}_{k}^{\nu}=\left\{x \in M^{m} \mid d\left(a_{k}, x\right) \leqq d\left(a_{i}, x\right), \quad i=1, \cdots, \mu\right\} .
\end{aligned}
$$

THEOREM. For each $p \in M^{m}$, let $\bar{\gamma}(p)$ be the intersection of all the sets $\bar{\gamma}_{\boldsymbol{k}}^{m}$ containing $p$. If $\eta$ is small enough, $\{\bar{\gamma}\}=\left\{\bar{\gamma}(p) \mid p \in M^{m}\right\}$ is a subdivision of $M^{m}$ into the closed cells of a complex.

Proof. Note first that if $i \neq k, d\left(a_{k}, x\right)=d\left(a_{i}, x\right)$ defines the normal bisecting $(\nu-1)$-plane $L_{k i}^{\nu-1}$ of the segment $a_{k} a_{i}$, and $d\left(a_{k}, x\right)<d\left(a_{i}, x\right)$ defines the half-space $H_{\boldsymbol{k} i}^{\nu-1}$ of $E^{v}$ bounded by $L_{\boldsymbol{k} i}^{\nu-1}$ and containing $a_{k}$. Thus $\bar{c}_{\boldsymbol{k}}^{\nu}$ is the closure of the open convex polyhedral $\nu$-cell

$$
c_{k}^{\nu}=\bigcap_{i \neq k} H_{k i}^{\nu}=\left\{x \in E^{\nu} \mid d\left(a_{k}, x\right)<d\left(a_{i}, x\right), i \neq k\right\},
$$

which may be of infinite diameter.

(B) The set $\bar{\gamma}_{\boldsymbol{k}}^{m}=\bar{c}_{\boldsymbol{k}}^{\nu} \cap M^{m}$ is on the interior $B^{\nu}\left(a_{k}, \eta\right)$ of the sphere $S^{\nu-1}\left(a_{k}, \eta\right)$ of radius $\eta$ about $a_{k}$.

For, by (A), each point of $M^{m}-\bar{B}^{v}\left(a_{k}, \eta\right)$ is closer to some $a_{i} \neq a_{k}$ than to $a_{k}$, and $c_{k}^{\nu}$ is the set of all points which are closer to $a_{k}$ than to any $a_{i} \neq a_{k}$.

The fact that $M^{m}$ is compact and of class $C^{2}$ implies that there exists a number $\rho>0$ so small that no $(\nu-1)$-sphere of radius $\rho$ tangent to $M^{m}$ encloses a point of $M^{m}$. The cell $c_{\boldsymbol{k}}^{\nu}$ therefore contains all points at distances $\leqq \rho$ from $a_{k}$ on the normal $n$-plane $N^{n}\left(a_{k}\right)$ to $M^{m}$ at $a_{k}$, since each such point is closer to $a_{k}$ than to any $a_{i} \neq a_{k}$.

(C) Hence, if $L_{k i}^{\nu-1}$ (defined above) intersects $\bar{\gamma}_{\boldsymbol{k}}^{m}$, then $L_{k i}^{\nu-1} \cap N^{n}\left(a_{k}\right)$ is either vacuous or at distance $>\rho$ from $a_{k}$.

\footnotetext{
1 This work was partly supported by NSF Grant G-14431.
} 
(D) We take $\eta<\rho / 2$ and so small that each intersection $\sigma^{m}\left(a_{k}, \eta\right)$ $=M^{m} \cap B^{v}\left(a_{k}, \eta\right)$ is an $m$-cell, each secant of which, hence each tangent line, makes an angle $\leqq \pi / 4$ with the tangent plane $T^{m}\left(a_{k}\right)$ at $a_{k}$. The projection $\bar{\sigma}^{m} \rightarrow T^{m}\left(a_{k}\right)$ is a homeomorphism.

A closed $(n+1)$-dimensional half-plane $\bar{H}^{n+1}$, with $N^{n}\left(a_{k}\right)$ for boundary, intersects $T^{m}\left(a_{k}\right)$ in a closed half-line $\tau^{1}=T^{m}\left(a_{k}\right) \cap \bar{H}^{n+1}$ from $a_{k}$, and it intersects $\bar{\sigma}^{m}\left(a_{k}, \eta\right)$ in a radial arc $\kappa$, whose projection on $T^{m}\left(a_{k}\right)$ is a segment of $\tau^{1}$.

(E) If $\kappa$ is a radial arc of $\sigma^{m}\left(a_{k}, \eta\right)$ which intersects the plane $L_{k i}^{\nu-1}$ of statement $(\mathrm{C})$ on $B^{\nu}\left(a^{\star}, \eta\right)$, then $\kappa \cap L_{\mathbf{k} i}^{\nu-1}$ is a single point, at which $\kappa$ crosses $L_{\mathbf{k} i}^{\nu-1}$.

For otherwise, $L_{k i}^{\nu-1}$ contains a line $L^{1}$ which is a tangent or a secant to $\kappa$. Since $L^{1}$ lies in $\bar{H}^{n+1}$, makes an angle less than $\pi / 4$ with $\tau^{1}$, and passes, by (B), within $\eta$ of $a_{k}$, it intersects $N^{n}\left(a_{k}\right)$ within distance $2^{1 / 2} \eta$ of $a_{k}$. But, $L^{1} \cap N^{n}\left(a_{k}\right) \subset L_{k i}^{\nu-1} \cap N^{n}\left(a_{k}\right)$ must be at distance $>\rho>2 \eta$ from $a_{k}$, by (C) and (D). This contradiction proves (E).

It now follows easily, using (B), that each radial segment of $\sigma^{m}\left(a_{k}, \eta\right)$ intersects $\bar{c}_{\boldsymbol{k}}^{\nu}-c_{\boldsymbol{k}}^{\nu}$ in a single point, so that $\gamma_{k}^{m}=\sigma^{m}\left(a_{k}, \eta\right) \cap c_{\boldsymbol{k}}^{\nu}$ is an $m$-cell. The proof of the theorem is easy to complete. The complex $\{\gamma\}$ can be simplicially subdivided into a $C^{r}$-triangulation of $M^{m}$.

(F) It is possible to extend this method to a noncompact $M^{m}$ and to a manifold with boundary. In the noncompact case, $\left(a_{1}, \cdots, a_{\mu}\right)$ is replaced by a countable set without limit point satisfying a density requirement as in (A), where $\eta$ is replaced by a suitable positive function on $M^{m}$.

(G) The method can be modified to treat the case where $M^{m}$ is of class $C^{1}$ or is subject only to an appropriate Lipschitz condition. Locally triangulable structures made up of differentiable or Lipschitz manifolds, suitably restricted, can also be thus triangulated.

We hope to publish such extensions elsewhere, along with theorems regarding isotopic deformations carrying the resulting complexes into polyhedra.

\section{REFERENCES}

1. S. S. Cairns, On the triangulation of regular loci, Ann. of Math. vol. 35 (1934) pp. 579-587.

2. - Triangulation of the manifold of class one, Bull. Amer. Math. Soc. vol. 41 (1935) pp. 549-552.

3. H. Freudenthal, Die Triangulation der differenzierbaren Mannigfaltigkeiten, Nederl. Akad. Wetensch. vol. 42 (1939) pp. 880-901.

4. J. H. C. Whitehead, On $C^{1}$-complexes, Ann. of Math. vol. 41 (1940) pp. 809-824.

5. H. Whitney, Geometric integration theory, Princeton University Press, 1957, Chapter IV, Part B.

UNIVERSITY OF ILLINOIS 